\title{
FACTORS INFLUENCING COMPLIANCE TO THERAPEUTIC REGIMEN AMONG PATIENTS WITH HYPERTENSION
}

\section{DHANYA R DHARAN, MOLY KT*}

Department of Medical Surgical Nursing, Amrita College of Nursing, Amrita University, Kochi, Kerala, India. Email: ktmoly@aims.amrita.edu Received: 30 June 2017, Revised and Accepted: 9 September 2017

\begin{abstract}
Objectives: The objectives of the study were to identify the level of compliance to therapeutic regimen, assess the factors promoting and interfering compliance, and to find the association between level of compliance and factors influencing it in patients with hypertension in a Tertiary Care Hospital,
\end{abstract} Kochi, with a view to develop an information booklet.

Methods: Nonprobability convenience sampling technique was used to collect data from 150 individuals attending outpatient departments. Data on compliance were assessed through interview using standardized Hill And Bone High Blood Pressure Compliance. Scale and factors promoting and interfering compliance were assessed using self-developed semi-structured questionnaire.

Results: Only 55 (36.7\%) had good compliance, while 52 (34.7\%) had average and 43 (28.7\%) had poor compliance to anti-hypertensive therapeutic regimen. The major factors promoting compliance were found as patient-prescriber relationship 146 (97.3\%), family support 133 (88.7\%), motivation 125 (83.3\%), communication with healthcare providers 122 (81.3\%), health literacy 104 (69.3\%), and patient satisfaction 75 (50\%). The factors interfering with compliance were lack of self-esteem 136 (90.7\%), long-term adherence 129 (86\%), misconceptions and erroneous beliefs $122(81.3 \%)$, cost of therapy and income 100 (80\%), forgetfulness 107 (71.3\%), difficulty in adjustment to dietary change 82 (54.7\%), and fear of side effects 70 (50\%). A significant association between the level of compliance and factors promoting and interfering with compliance to therapeutic regimen $(\mathrm{p}<0.01)$ was noted. Level of compliance with therapeutic regimen was found to be lower in patients with associated comorbidities such as diabetes mellitus $\left(\chi^{2}=9.52, \mathrm{p}<0.01\right)$ and coronary artery disease $\left(\chi^{2}=6.737, \mathrm{p}<0.05\right)$.

Conclusion: The study concludes the significance of developing systems to tack and ensure compliance to therapy among hypertensives with a focus on factors promoting compliance not only from the patient perspective but also from the perspective of family and society.

Keywords: Hypertension, Compliance, Therapeutic regimen, Factors influencing.

(c) 2017 The Authors. Published by Innovare Academic Sciences Pvt Ltd. This is an open access article under the CC BY license (http://creativecommons. org/licenses/by/4. 0/) DOI: http://dx.doi.org/10.22159/ajpcr.2017.v10i12.21034

\section{INTRODUCTION}

Hypertension is a major medical and public health problem. High blood pressure is the most prevailing cardiovascular risk factor worldwide. Recent report indicated that nearly 1 billion adults had hypertension in 2000 , and this is predicted to increase to 1.56 billion by 2025 [1].

The prevalence of hypertension in Indians is $25 \%$ in urban and $10 \%$ in rural population. There are nearly 31.5 million hypertensives in rural and 34 million in urban populations. Hypertension is directly accountable for $57 \%$ of stroke deaths and $24 \%$ of coronary artery disease deaths in India [2].

In a study carried out by Gupta et al. [3], among women from four urban and five rural areas (sample size - 4606) in India, found that there is a high prevalence of hypertension in middle-aged Asian Indian women. Very low awareness, treatment, and control status were observed.

Despite the effectiveness of anti-hypertensive treatment, high blood pressure is rarely controlled and it can result in a huge adverse impact on quality of life [4]. The primary goals in anti-hypertensive therapy include achieving and maintaining a controlled blood pressure, reducing cardio vascular risk and target organ diseases. Lifestyle modifications include weight reduction, dietary approaches to stop hypertension eating plan, dietary sodium restriction, moderation of alcohol consumption, regular physical activity, avoidance of tobacco use, and management of psycho-socio-risk factors [5].

Although safe and effective drugs are expected to be available, the management of hypertension is still far from optimum, especially in the developing countries. A major factor accounting for inadequate treatment of hypertension is poor compliance [6].

Side effects of anti-hypertensive drugs are common and may be so severe or undesirable that the patient does not comply with the therapy $[7,8]$. Patient and caregiver teaching, changing the drug or decreasing the dosage may help the patient adhere to therapy. Sexual problems with many of the anti-hypertensive drugs can be a major reason for nonadherence. It is important to help the patient and care giver understand that hypertension is a chronic illness that cannot be cured. The fact that it can be controlled with drug therapy, dietary changes, physical activity, periodic follow-up, and other relevant lifestyle modifications need to be emphasized for better compliance.

A descriptive study to identify factors influencing compliance with therapeutic regimen among patients with hypertension was conducted in selected outpatient departments (OPDs) of a Tertiary Care Hospital, Kochi. The purpose of the study was to enhance compliance by preparing an information booklet after exploring the current level of compliance and factors promoting and interfering with compliance to therapeutic regimen for patients with hypertension.

\section{METHODS}

A nonexperimental descriptive study on 150 hypertensives on medication for 1 year and above, who were attending general medicine OPD of a Tertiary Care Hospital was conducted. Individuals were taken using nonprobability convenience sampling technique. Patients with hearing impairment/mentally challenged/neurologically debilitated were excluded from the study. 


\section{Data collection instruments}

- Tool 1: Structured interview schedule to assess sociodemographic and clinical variables: Sociodemographic variables included gender, age, education, monthly income, and area of residence. Clinical variables included duration since diagnosis, current medications, frequency of drug intake, and number of anti-hypertensive drugs per day.

- Tool 2: Standardized Hill Bone Compliance Scale: It was used to assess the level of compliance with the anti-hypertensive regimen. The tool focused on three domains: Medication intake, reduced sodium intake, and appointment keeping. This is a four-point scale with a minimum score of 14 and a maximum score of 56 . A score of 14-28 is interpreted as good compliance, 29-42 as average, and 43-56 as poor compliance. Permission was obtained from Hill M N, Bone L R, Kim M T for using the scale.

- Tool 3: Semi-structured questionnaire on factors influencing compliance and noncompliance with therapeutic regimen among patients with hypertension: It had 39 questions distributed under seven areas such as social and economic factors (5 questions), psychological factors (7 questions), cultural beliefs and attitude (7 questions), treatment centered factors (6 questions), healthcare system-related factors (6 questions), patient-centered factors ( 9 questions), and other factors felt by the patient. The reliability was assessed using the split-half technique and was found to be 0.8 . The content validity index was 1 .

\section{Data collection}

Based on the inclusion criteria, individuals were selected using convenience sampling. Informed consent was obtained from each patient. All the data except the clinical variable were collected using interview technique. Data on clinical variables were collected from patient's medical records.

\section{Data analysis}

Data were analyzed in frequency, percentage, and Chi-square using SPSS version 17.

\section{RESULTS}

\section{Section I: Sample characteristics}

Table 1 shows that 124 (82.7\%) individuals were between the age group of 40 and 70 years. The majority were males, i.e., 79 (52.7\%). $123(82 \%)$ were having only primary/secondary education and $9(6 \%)$ were illiterate. $118(78.7 \%)$ had only a monthly income of $<10,000 /$. Majority of them resides in rural areas 112 (74.7\%).

Section II: Compliance to therapeutic regimen among hypertensives $55(36.7 \%)$ had good, $52(34.7 \%)$ had average and $43(28.6 \%)$ had poor compliance to anti hypertensive therapeutic regimen. Even though the percentage of poor compliance is $43(28.6 \%)$ it is significant when considering the life threatening complications associated with hypertension (Fig. 1).

Section III: Factors promoting and interfering with compliance to therapeutic regimen in hypertensives

The major factors promoting compliance to therapeutic regimen were found as patient prescriber relationship 146 (97.3\%), family support 133 (88.7\%), motivation $125(83.3 \%)$, communication with health care providers $122(81.3 \%)$, health literacy $104(69.3 \%)$ and patient satisfaction 75 (50\%) (Fig. 2).

The factors interfering with compliance to therapeutic regimen were lack of self esteem 136 (90.7\%), long term adherence to therapeutic regimen129 (86\%), misconceptions and erroneous beliefs 122 (81.3\%), cost of therapy and income $100(80 \%)$, forgetfulness 107 (71.3\%), difficulty in adjustment to dietary change $82(54.7 \%)$ and fear of side effects 70 (50\%) (Fig. 3).

\section{DISCUSSION}

The study provides evidence that compliance level varies in hypertensives. The level of compliance was poor in 43 (28.6\%) of patients which make
Table 1: Distribution of individuals based on demographic variables $(n=150)$

\begin{tabular}{lc}
\hline Demographic variables & Frequency (\%) \\
\hline Gender & \\
Male & $79(52.7)$ \\
Female & $71(47.3)$ \\
Age (in years) & \\
$21-30$ & $1(0.6)$ \\
$31-40$ & $12(8.0)$ \\
$41-50$ & $37(24.7)$ \\
$51-60$ & $46(30.7)$ \\
$61-70$ & $41(27.3)$ \\
$>71$ & $13(8.7)$ \\
Education & \\
Illiterate & $9(6.0)$ \\
Primary & $51(34.0)$ \\
Secondary & $46(30.7)$ \\
Higher secondary & $26(17.3)$ \\
Graduate & $14(9.3)$ \\
Postgraduate & $3(2.0)$ \\
Postgraduate and above & $1(0.7)$ \\
Monthly income (in rupees) & \\
$<5000$ & $70(46.7)$ \\
$5001-10000$ & $48(32.0)$ \\
10001-15000 & $17(11.3)$ \\
15001-25000 & $7(4.7)$ \\
25001-35000 & $5(3.3)$ \\
>35000 & $3(2.0)$ \\
Area of residence & \\
Rural & $112(74.7)$ \\
Urban & $38(25.3)$ \\
\hline
\end{tabular}

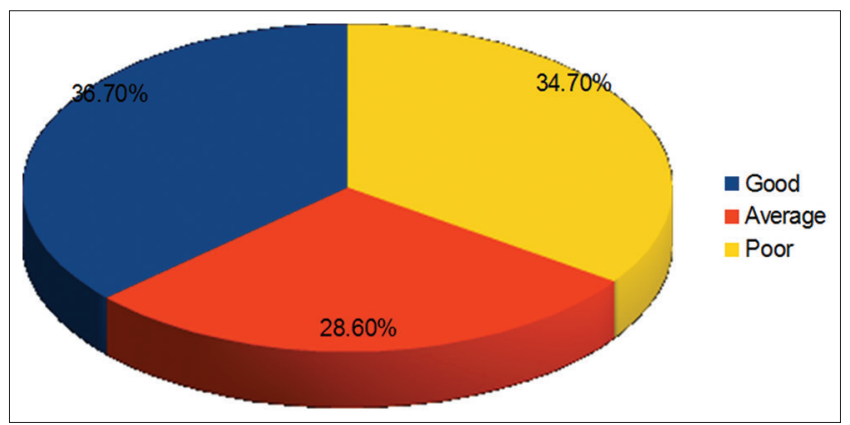

Fig. 1: Pie diagram showing level of compliance among patients with hypertension

it clear that they are at risk of developing life-threatening complications leading to death. Hence, it is significant. Similar findings were observed in the study conducted by Amira and Okubadejo [9] in which it was found that $45.5 \%$ had good, $34.2 \%$ had average, and $27.3 \%$ had poor compliance with therapeutic regimen. They also found that blood pressure was significantly better among compliant (45.9\%) than noncompliant patients $(27.3 \%)\left(\chi^{2}=7.35 \mathrm{p}=0.007\right)$ which was not assessed in the study. In a cross-sectional study conducted by Sowielem and Elzubier [10], good compliance was found in $34.2 \%$, average compliance in $37.8 \%$, and poor compliance in $28 \%$ of patients.

Lim and Ngah [11] interviewed 60 hypertensives admitted to medical wards to determine reasons for their previous drop out from treatment in Singapore. The reasons for drop out were as follows: $95 \%$ of the patients were unaware about the long-term adherence to therapeutic regimen, $85 \%$ of the patients were lacking in motivation, $78 \%$ equated relief of symptoms such as headache, dizziness with control of hypertension, $72 \%$ complained of long waiting time required to obtain care, $60 \%$ lack of health literacy, and $52 \%$ fear the side effects of drug. They found that patient's beliefs about the causes and meaning of illness and motivation to follow the therapy were strongly related to 


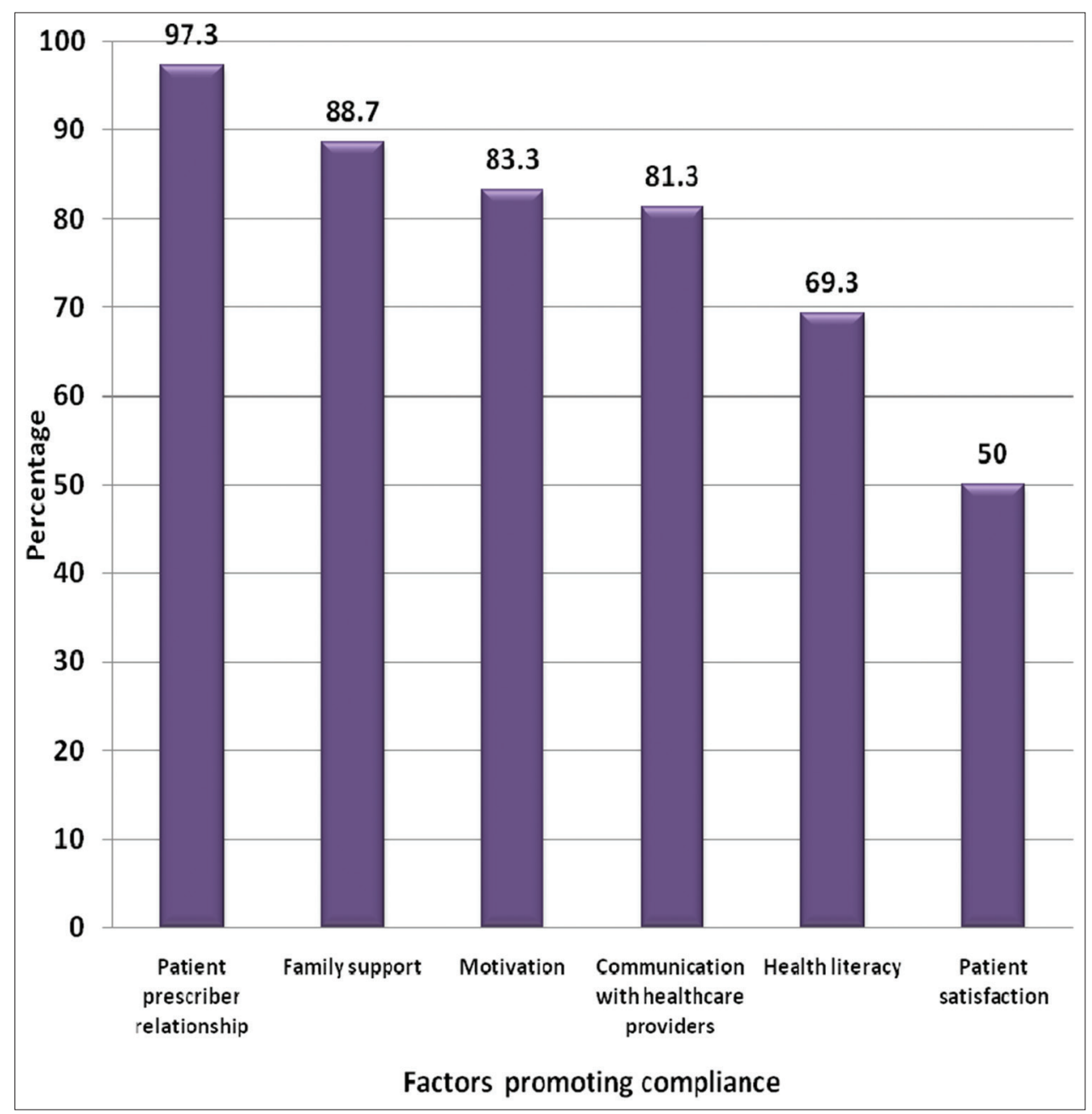

Fig. 2: Factors promoting compliance to therapeutic regimen in hypertensives

their compliance with healthcare. This study supports the findings of the present study.

The cost of therapy and income $(80 \%)$ interfering with compliance found in this study emphasize the study findings of Lekshmi et al. Manna that the medication availability and affordability for hypertension particularly in the public sector need improvement [12].

In the study of Thakur et al. [13] on health awareness and treatment compliance of hypertension, among 72 women at Chandigarh in 2009, the main reasons for discontinuing the treatment were found to be ignorance about the need for regular treatment (33.3\%), high cost of medicines (19.44\%), and nonavailability of a family member who can go with the patient to hospital (9.72\%). Only $16.7 \%$ were found to be compliant with the therapeutic regimen.

A significant association was observed between level of compliance and factors influencing compliance and noncompliance to therapeutic regimen $(\mathrm{p}<0.01)$. The level of compliance was lower among individuals with associated comorbidities such as diabetes $\left(\chi^{2}=9.52, \mathrm{p}<0.01\right)$, or coronary artery disease $\left(\chi^{2}=6.737, \mathrm{p}<0.05\right)$. Similar finding was reported in the study conducted at Saudi Arabia by Abuabker Ibrahim Elbur where comorbidity was found to be an important factor in the commitment to a healthy diet $(\mathrm{p}=0.012)$, regular exercise $(\mathrm{p}=0.028)$, and medication $(\mathrm{p}=0.002)[14]$.

Level of compliance was found to be better among retired people than employed $\left(\chi^{2}=17.223, p<0.01\right)$. Married people had a relatively higher level of compliance than unmarried $\left(\chi^{2}=16.3, \mathrm{p}<0.01\right)$. Nonsmokers were at a higher level of compliance $\left(\chi^{2}=6.822, p<0.05\right) .147(98 \%)$ individuals had hypertension for more than 6 years. Among the antihypertensive drug regimen, calcium channel blockers 74 (49.3\%) were the most commonly prescribed drug.

One of the limitations of the study was that the Hill Bone High Blood Pressure Compliance Scale used in the present study focused only on three domains, i.e., medication intake, reduced sodium intake, and appointment keeping. Hence, other dimensions of adherence require further investigation.

The study findings on factors promoting and interfering with compliance once again emphasize patient and healthcare provider relationship, family support, motivation, the need to eliminate misconceptions, fear of side effects, and providing adequate information on the need for long-term adherence to therapy.

\section{CONCLUSION}

The researcher concludes that noncompliance with hypertension although found only in one-third of the sample studied is a significant threat considering the complications of the disease. The chronic nature and silent symptoms associated with the disease and the very multidimensional nature of the phenomenon of adherence makes it more difficult. It is under the responsibility of the healthcare providers to give them the necessary information. This was done by preparing an information booklet by the investigator who was sent to the study individuals. 


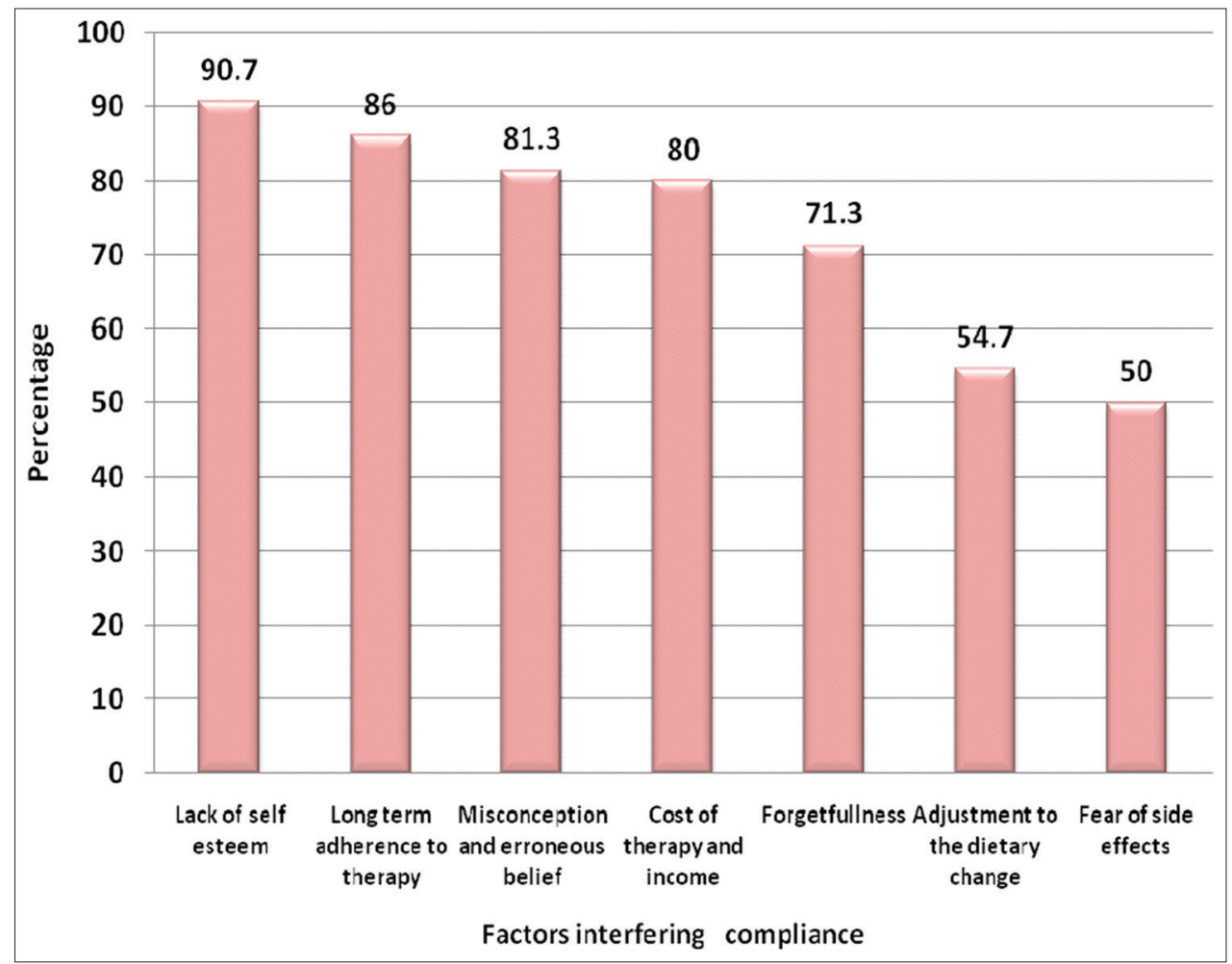

Fig. 3: Factors interfering with compliance to therapeutic regimen in hypertensives

\section{Ethical clearance}

Ethical clearance was obtained from the Research Committee of Amrita College of Nursing and Thesis Review Committee of the Institution. Consent was taken from each individual before data collection.

\section{REFERENCES}

1. Sruthy PN, Anoop KR. Healing hypertension: A focus on alternative systems of medicine. Int J Pharm Sci Rev Res 2013;21:264-73.

2. Gupta R. Trends in hypertension epidemiology in India. J Hum Hypertens 2004;18(2):73-8.

3. Gupta R, Pandey RM, Misra A, Agrawal A, Misra P, Dey S, et al. High prevalence and low awareness, treatment and control of hypertension in Asian Indian women. J Hum Hypertens 2012;26(10):585-93.

4. Shahina PT, Ga RK, Rb K, Ab JV, Ka SV. The impact of pharmacist interventions on quality of life in patients with hypertension. Int $\mathrm{J}$ Pharm Sci Rev Res 2010;5:172-6.

5. Lewis LS, Dirksen SR, Heitkemper MM, Bucher L. Lewis Medical Surgical Nursing. $2^{\text {nd }}$ ed. New Delhi: Elsevier; 2014.

6. WHO. Cardiovascular Disease. Geneva: WHO; 2012. p. 210-8

7. Aronow WS, Fleg JL, Pepine CJ, Artinian NT, Bakris G, Brown AS, et al. Expert consensus document on hypertension in the elderly. J Am Coll Cardiol 2011;123(21):2434-506.

8. Olson J. Clinical Pharmacology Made Ridiculously Simple. $4^{\text {th }}$ ed. Miami: Ed Master; 2011

9. Amira CO, Okubadejo NU. Factors influencing non-compliance with anti-hypertensive drug therapy in Nigerians. Niger Postgrad Med J 2007;14(4):325-9.

10. Sowielem A, Elzubier AG. Compliance and knowledge of hypertensive patients attending PHC. East Mediterr Health J 2008;4:301-7.

11. Lim TO, Ngah BA. The Mentakab hypertension study project. Part IIWhy do hypertensives drop out of treatment? Singapore Med J 1991;32(4):249-51.

12. Lekshmi SG, Mohanta P, Revikumar KG, Manna PK. Burden of therapy in patients suffering from diabetes mellitus and hypertension. Int J Pharm Pharm Sci 2017;9(5):364-9.

13. Thakur K, Malhotra P, Walia I, Kumar R. Health awareness and treatment compliance of high blood pressure among women in a peri-urban colony of Chandigarh, India. J Indian Med Assoc 1999;97(6):217-9.

14. Abuabker IE. Level of adherence to lifestyle changes and medications among male hypertensive patients in two hospitals in Taif; Kingdom of Saudi Arabia. Int J Pharm Pharm Sci 2015;7(4):168-72. 